\title{
Nonclassicality in weak measurements
}

\author{
Lars M. Johansen ${ }^{1, *}$ and Alfredo Luis ${ }^{2, \dagger}$ \\ ${ }^{1}$ Department of Technology, Buskerud University College, N-3601 Kongsberg, Norway \\ ${ }^{2}$ Departamento de Óptica, Facultad de Ciencias Físicas, Universidad Complutense, 28040 Madrid, Spain
}

(Received 5 August 2004; published 29 November 2004)

\begin{abstract}
We examine weak measurements of arbitrary observables where the object is prepared in a mixed state and on which measurements with imperfect detectors are made. The weak value of an observable can be expressed as a conditional expectation value over an infinite class of different generalized Kirkwood quasiprobability distributions. "Strange" weak values for which the real part exceeds the eigenvalue spectrum of the observable can only be found if the Terletsky-Margenau-Hill distribution is negative or, equivalently, if the real part of the weak value of the density operator is negative. We find that a classical model of a weak measurement exists whenever the Terletsky-Margenau-Hill representation of the observable equals the classical representation of the observable and the Terletsky-Margenau-Hill distribution is non-negative. Strange weak values alone are not sufficient to obtain a contradiction with classical models. We propose feasible weak measurements of photon number of the radiation field. Negative weak values of energy contradict all classical stochastic models, whereas negative weak values of photon number contradict all classical stochastic models where the energy is bounded from below by the zero-point energy. We examine coherent states in particular and find negative weak values with probabilities of $16 \%$ for kinetic energy (or squared field quadrature), $8 \%$ for harmonic oscillator energy, and $50 \%$ for photon number. These experiments are robust against detector inefficiency and thermal noise.
\end{abstract}

DOI: 10.1103/PhysRevA.70.052115

PACS number(s): 03.65.Ta, 42.50.Dv

\section{INTRODUCTION}

Physics is an endeavor to construct a mathematical model of nature. The basic mathematical model of classical physics is one in which all dynamical variables have definite values simultaneously. With the advent of statistical mechanics, a probabilistic description was introduced in which each dynamical variable would have some value with a certain probability. This is the most general description that classical physics can provide. A great variety of phenomena can be explained in terms of such a model. For example, most optical phenomena can be described in terms of a model of complex, stochastic amplitudes. It was not until 1977 that this model was found to break down in an experiment which demonstrated the phenomenon of antibunching [1].

In this paper we examine a relatively new type of measurement known as weak measurements [2]. Weak measurements may be performed in exactly the same way as standard von Neumann measurements, but with a weakened interaction [3]. In a weak measurement, the pointer will on average register the expectation value of the observable that is measured. However, when the weak measurement is conditioned on a second postselection measurement, the pointer registers the real part of what is known as the "weak value" of the observable. These weak values have caused a lot of controversy, in particular because they may exceed the eigenvalue spectrum of the observable.

The main objective of this paper is to discuss the conditions under which a classical description of weak measure-

\footnotetext{
*Electronic address: lars.m.johansen@hibu.no

${ }^{\dagger}$ Electronic address: alluis@ fis.ucm.es
}

ments is possible, taking into account practical experimental limitations and possibilities. In particular, our purpose is to investigate in further detail the failure of providing a classical description of coherent states in such experiments and to propose a feasible experiment demonstrating nonclassical properties of coherent states.

In Sec. II we give a brief review of the Glauber classicality criterion and discuss its possible limitations.

In Sec. III and Appendixes A and B we consider a general class of weak measurements where the object may be prepared in a mixed state and where the pointer may be prepared in an arbitrary mixed state of vanishing current density. We consider detectors of arbitrary quantum efficiency and derive a generalized weak value.

In Sec. IV we demonstrate that the weak value can be expressed as a conditional expectation over an infinite set of different quasiprobability distributions. These distributions can be regarded as generalizations of the complex Kirkwood distribution [4] or the standard ordered distribution [5]. We denote such distributions by $S$ distributions. The TerletskyMargenau-Hill distribution [6,7], or $T$ distribution, is the real parts of the $S$ distribution. We find that strange weak values (for which the real part exceeds the eigenvalue spectrum of the observable) can only exist if the $T$ distribution takes negative values. We demonstrate that a classical model of a weak measurement exists whenever the representation of the observable coincides with the classical representation of the observable and the $T$ distribution is non-negative. We demonstrate in particular that negative weak values of energy contradict all classical stochastic models and that negative weak values of photon number contradict a stochastic model where the energy is bounded from below by the zero-point energy. 
In Sec. V we propose two feasible practical realizations of weak measurements of photon number and energy.

In Sec. VI we consider in particular weak measurements on coherent states. We demonstrate that coherent states display negative weak values with probabilities of $16 \%$ for kinetic energy (or squared field quadrature), $8 \%$ for harmonic oscillator energy, and $50 \%$ for photon number. We find that these effects are robust against detector inefficiency and thermal noise.

\section{GLAUBER CLASSICALITY CRITERION}

Glauber and Sudarshan have demonstrated that any density operator can be expanded diagonally in terms of coherent states $[8,9]$

$$
\hat{\rho}=\int d^{2} \alpha P(\alpha)|\alpha\rangle\langle\alpha| .
$$

The weight function $P(\alpha)$ is known as the $P$ distribution. Furthermore, one may write the expectation value of any normal ordered operator $\mathcal{N}\left[\hat{O}\left(\hat{a}^{\dagger}, \hat{a}\right)\right]$ as an integral of the form

$$
\left\langle\mathcal{N}\left[\hat{O}\left(\hat{a}^{\dagger}, \hat{a}\right)\right]\right\rangle=\int d^{2} \alpha O\left(\alpha^{*}, \alpha\right) P(\alpha),
$$

where $a^{*}$ is the $c$-number representation of $\hat{a}^{\dagger}$ and $a$ is the $c$-number representation of $\hat{a}$. Therefore, if the $P$ distribution has the properties of a valid probability distribution, one may say that a classical representation exists for any normal ordered operator product. This is known as the "optical equivalence theorem" [9].

The optical equivalence theorem is the basis of the Glauber classicality criterion, according to which all states for which the $P$ distribution is a probability distribution are regarded as essentially classical. If $P$ fails to be a probability distribution, the state is considered as nonclassical $[8,10-13]$. The Glauber criterion is widely accepted as giving the most general distinction between quantum and classical states. It is the basis of various measures of "nonclassicality." For example, Hillery has defined a measure of nonclassicality based on the distance in Hilbert space between the object state and coherent states [14]. Lee has defined a nonclassical depth defined as the minimum average number of thermal photons that must be added to render the $P$ distribution nonnegative [15]. More recently, Richter and Vogel have derived a hierarchy of observable conditions to test the Glauber criterion $[16,17]$.

The Glauber criterion must be considered a conjecture rather than a proven theorem. It is based on some plausible arguments. First, due to the multitude of arguments in favor of coherent states as the only classical-like pure states, it is natural to assume that also classical mixtures of coherent states are classical. This is in fact equivalent to Glauber's classicality criterion, as can be seen from Eq. (1). If the $P$ distribution is a probability distribution, the density matrix can be expressed as a classical mixture of coherent states. Second, a non-negative $P$ distribution ensures that the whole range of $s$-ordered Wigner distributions is also non-negative.
Therefore, the $P$ distribution is most "sensitive" to nonclassicality among all $s$-ordered distributions.

It is puzzling that a definition of nonclassicality depends on the ability to represent expectations of normally ordered operator expressions as classical expectation values over a probability distribution. This is sometimes attributed to the fact that normal ordering of operators is closely associated to the theory of photodetection.

However, we may devise experimental procedures related to operator orderings different from normal ordering of annihilation and creation operators, which may display clear contradictions between the classical and quantum descriptions for the same experiment. This is actually the case of weak measurements, whose statistics are related to $T$ distributions instead of to the more standard $s$-ordered distributions. We show below that weak measurements lead to strange outputs provided that the $T$ distribution takes negative values. Moreover, we will show in this paper that a classical stochastic model may fail to describe weak measurements even when the $P$ distribution is a non-negative probability distribution.

\section{WEAK MEASUREMENTS}

A "measurement" comes about when an auxiliary system interacts with an object. By examining the properties of the auxiliary system after the interaction, it may be possible to extract information about the object. The auxiliary system is frequently called a "measurement apparatus" or a "pointer system." The basic theory of quantum measurement was examined by von Neumann in his seminal work on the mathematical foundations of quantum mechanics [18]. In this work, von Neumann represented the interaction between the object and pointer by an interaction Hamiltonian of the form (throughout we will use units in which $\hbar=1$ )

$$
\hat{H}_{\epsilon}=\epsilon \delta(t) \hat{\nu} \otimes \hat{P} .
$$

A short explanation of the terms involved is in place. The constant $\epsilon$ represents the interaction strength. The interaction is of short duration, represented in idealized form by the $\delta$ term. The Hermitian observable $\hat{\nu}$ belongs to the object Hilbert system $\mathcal{H}_{s}$ and is the observable that we want to "measure." The observable $\hat{P}$ is the pointer momentum and belongs to the pointer Hilbert space $\mathcal{H}_{a}$. Although seemingly artificially constructed, this interaction model has served as an archetype of the interaction mechanism in quantum measurements. It has been found that the conclusions that can be drawn from this model are generic to a number of other interaction mechanisms (for a closer discussion of the specific properties of this interaction Hamiltonian, see Ref. [19]).

In a standard, projective measurement, the pointer position $\hat{Q}$, with $[\hat{Q}, \hat{P}]=i$, displays one of the eigenvalues of the object observable $\hat{\nu}$ after the measurement interaction. It was demonstrated by von Neumann that in order to accomplish this, the state of the pointer prior to the interaction should have a small position spread [18]. The same effect can be accomplished by allowing the interaction strength $\epsilon$ to be 
sufficiently large [19]. For this reason, this type of measurement is frequently called a "strong measurement."

Until recently, it was thought that strong measurements are the only useful type of measurements in quantum mechanics. However, in 1988 Aharonov et al. proposed a new type of measurement that they called "weak measurements" [2]. Such measurements also employ the von Neumann interaction mechanism (3), the difference being that the pointer is assumed to be in an initial state of large position uncertainty. More specifically, they assumed that the initial state of the pointer was a Gaussian with large spread.

Recently, it was shown that weak measurements can be performed also when the pointer is in an arbitrary mixed state, provided that the interaction strength $\epsilon$ is sufficiently small and the current density of the pointer vanishes [3]. This description can be generalized further by taking into account finite efficiency of the detectors. A detector of finite efficiency can be represented by positive-operator-valued measure (POVM) (see Appendix A). In this case, the weak value of the observable $\hat{v}$ conditioned on an imperfect postselection of the observable $\hat{\phi}$ on the object is (see Appendix B)

$$
\nu_{w}(\phi)=\frac{\operatorname{Tr}\left(\hat{\Pi}_{\phi} \hat{\nu} \hat{\rho}_{s}\right)}{\operatorname{Tr}\left(\hat{\Pi}_{\phi} \hat{\rho}_{s}\right)},
$$

where $\hat{\Pi}_{\phi}$ is a diagonal POVM representing the imperfect postselection and $\hat{\rho}_{s}$ is the initial state of the object [see Eq. (B5)].

It has been noted that weak values are conditional expectation values of a complex quasiprobability distribution $[20,21]$. Furthermore, weak values have been examined in other contexts than under weak measurements. For example, they are sometimes called "local values" [22]. The relation between such local values and quasiprobability distributions has been examined in several settings [23-25]. The weak value of an observable is also the optimal estimator of the observable between preselection and postselection [26]. The relation between the optimal estimator of an observable and phase-space distributions was examined in [27].

\section{CLASSICAL MODELS OF WEAK MEASUREMENTS}

Under what circumstances is it possible to find a classical representation of a weak measurement? Or put differently, under what circumstances can the outcome of a weak measurement be modeled in terms of a classical, stochastic model? By answering this question, we will also understand under what circumstances weak measurements demonstrate nonclassical properties of the quantum state under consideration.

We begin by inserting the definition (B5) for the diagonal postselection POVM into Eq. (4). We then have

$$
\nu_{w}(\phi)=\frac{\operatorname{Tr}\left[\int d \phi^{\prime} \Pi_{\phi}\left(\phi^{\prime}\right)\left|\phi^{\prime}\right\rangle\left\langle\phi^{\prime}\right| \hat{\nu} \hat{\rho}_{s}\right]}{\operatorname{Tr}\left[\int d \phi^{\prime} \Pi_{\phi}\left(\phi^{\prime}\right)\left|\phi^{\prime}\right\rangle\left\langle\phi^{\prime}\right| \hat{\rho}_{s}\right]} .
$$

By performing the trace over any complete set of states we obtain

$$
\nu_{w}(\phi)=\frac{\int d \phi^{\prime} \Pi_{\phi}\left(\phi^{\prime}\right)\left\langle\phi^{\prime}\left|\hat{\nu} \hat{\rho}_{s}\right| \phi^{\prime}\right\rangle}{\int d \phi^{\prime} \Pi_{\phi}\left(\phi^{\prime}\right)\left\langle\phi^{\prime}\left|\hat{\rho}_{s}\right| \phi^{\prime}\right\rangle} .
$$

We employ an arbitrary complete set of states $|\xi\rangle$ :

$$
\int d \xi|\xi\rangle\langle\xi|=\mathrm{I} .
$$

We may then write Eq. (6) in the form

$$
\nu_{w}(\phi)=\frac{\int d \xi d \phi^{\prime} \Pi_{\phi}\left(\phi^{\prime}\right) S_{\hat{\nu}}\left(\phi^{\prime}, \xi\right) S\left(\phi^{\prime}, \xi\right)}{\int d \xi d \phi^{\prime} \Pi_{\phi}\left(\phi^{\prime}\right) S\left(\phi^{\prime}, \xi\right)},
$$

where

$$
S_{\hat{\nu}}(\phi, \xi)=\frac{\langle\phi|\hat{\nu}| \xi\rangle}{\langle\phi \mid \xi\rangle}
$$

is a $c$-number representation of the observable $\hat{\nu}$. In fact, it is the weak value for the observable preselected in the state $|\xi\rangle$ and postselected in the state $|\phi\rangle$. Also,

$$
S(\phi, \xi)=\langle\phi \mid \xi\rangle\left\langle\xi\left|\hat{\rho}_{s}\right| \phi\right\rangle
$$

is a generalization of the Kirkwood distribution for arbitrary observables $\hat{\xi}$ and $\hat{\phi}$ [4]. The Kirkwood distribution is also known as the antistandard ordered distribution [5]. $S$ is also a generalization of the standard ordered distribution, which is the complex conjugate of the Kirkwood distribution [5]. In this paper, we will simply denote it by the $S$ distribution, and we will refer to $S_{\hat{\nu}}$ as the $S$ representation of the observable $\hat{\nu}$.

The $S$ distributions are in general complex and as such are quasiprobability distributions. Nevertheless, they possess some of the properties of classical joint distributions. For example, assuming that both eigenstates $|\phi\rangle$ and $|\xi\rangle$ constitute complete sets, it is straightforward to show that they yield correct marginal distributions when integrated over either variable:

$$
\begin{aligned}
& \left\langle\phi\left|\hat{\rho}_{S}\right| \phi\right\rangle=\int d \xi S(\phi, \xi), \\
& \left\langle\xi\left|\hat{\rho}_{s}\right| \xi\right\rangle=\int d \phi S(\phi, \xi) .
\end{aligned}
$$

It is straightforward to show that also the complex conjugate distribution $S^{*}(\phi, \xi)$ fulfills such marginality conditions.

The $S$ distribution can be expressed in the form

$$
S(\phi, \xi)=|\langle\phi \mid \xi\rangle|^{2} \frac{\left\langle\xi\left|\hat{\rho}_{s}\right| \phi\right\rangle}{\langle\xi \mid \phi\rangle} .
$$

In this form, the $S$ distribution is a product of a non-negative probability distribution and the weak value of the density operator. If $|\xi\rangle$ or $|\phi\rangle$ are eigenstates of the density operator, $S$ will be real and non-negative. 
Classically, the weak value of an observable is the conditional expectation of that observable [3]. Expression (8) demonstrates that there exists an infinite set of representations under which the weak value can be expressed as a conditional expectation of a $c$-number variable $S_{\hat{v}}$ over an $S$ distribution. For each choice of basis $|\xi\rangle$, a different $S$ distribution is obtained. However, some of these representations bear little resemblance to any classical model. For example, in some models the $S$ representation of the Hermitian observable $\hat{v}$ is complex. As a basic requirement on a classical model, we shall in the following restrict our attention to the subset of representations for which Hermitian observables have real representations:

$$
\operatorname{Im} S_{\hat{\nu}}(\phi, \xi)=0 .
$$

We also introduce the $T$ representation of $\hat{\nu}$ and the $T$ distribution $[6,7]$ :

$$
\begin{aligned}
T_{\hat{\nu}}(\phi, \xi) & =\operatorname{Re} S_{\hat{\nu}}(\phi, \xi), \\
T(\phi, \xi) & =\operatorname{Re} S(\phi, \xi) .
\end{aligned}
$$

It is straightforward to show that also these provide correct marginal distributions

$$
\begin{aligned}
& \left\langle\phi\left|\hat{\rho}_{s}\right| \phi\right\rangle=\int d \xi T(\phi, \xi), \\
& \left\langle\xi\left|\hat{\rho}_{s}\right| \xi\right\rangle=\int d \phi T(\phi, \xi) .
\end{aligned}
$$

Because of the classicality condition (14), the $S$ and $T$ representations of the observable are the same:

$$
T_{\hat{\nu}}(\phi, \xi)=\frac{\langle\phi|\hat{\nu}| \xi\rangle}{\langle\phi \mid \xi\rangle} .
$$

The distribution of the postselection observable $\hat{\phi}$, taking into account the finite detector efficiency represented by $\Pi_{\phi}$, can be found from both the complex $S$ distribution and the real $T$ distribution through the integrals

$$
\begin{aligned}
\operatorname{Tr}\left(\Pi_{\phi} \hat{\rho}_{s}\right) & =\int d \xi d \phi^{\prime} \Pi_{\phi}\left(\phi^{\prime}\right) S\left(\phi^{\prime}, \xi\right) \\
& =\int d \xi d \phi^{\prime} \Pi_{\phi}\left(\phi^{\prime}\right) T\left(\phi^{\prime}, \xi\right) .
\end{aligned}
$$

In a weak measurement, the real part of the weak value $\nu_{w}$ is registered by the pointer. Under the assumption (14), we may write

$$
\operatorname{Re}\left(\nu_{w}\right)=\frac{\int d \xi d \phi^{\prime} \Pi_{\phi}\left(\phi^{\prime}\right) T_{\hat{\nu}}\left(\phi^{\prime}, \xi\right) T\left(\phi^{\prime}, \xi\right)}{\int d \xi d \phi^{\prime} \Pi_{\phi}\left(\phi^{\prime}\right) T\left(\phi^{\prime}, \xi\right)} .
$$

This expression, which reflects the expectation of the pointer displacement in a weak measurement, bears a formal resemblance to a classical conditional expectation. The difference is that the $T$ distribution may take negative values and that the $T$ representation of the observable may differ strongly from the representation of the observable in classical theory. A classical representation of the pointer displacement can be said to exist if $T_{\hat{v}}$ equals the classical representation of the observable and if also the $T$ distribution is non-negative.

What determines the sign of the $T$ distribution? From Eqs. (13) and (16) it follows that

$$
T(\phi, \xi)=|\langle\phi \mid \xi\rangle|{ }^{2} \operatorname{Re}\left(\frac{\left\langle\xi\left|\hat{\rho}_{s}\right| \phi\right\rangle}{\langle\xi \mid \phi\rangle}\right) .
$$

Thus, the sign of the $T$ distribution equals the sign of the real part of the weak value of the density operator. Therefore, a requirement for nonclassicality is that the real part of the weak value of the density operator should be negative.

In the following, we consider two representations that may provide classical-like models of weak measurements. The first representation, which we shall call the eigenvalue representation, is found when the basis $|\xi\rangle$ is chosen as eigenstates of the observable $\hat{\nu}$. In this representation the $S$ and $T$ representations of the observable $\hat{\nu}$ are the eigenvalues $\nu$ :

$$
S_{\hat{\nu}}(\phi, \nu)=T_{\hat{\nu}}(\phi, \nu)=\nu .
$$

By using this representation, we have

$$
\begin{gathered}
\nu_{w}(\phi)=\int d \nu \nu S_{\eta}(\nu \mid \phi), \\
\operatorname{Re} \nu_{w}(\phi)=\int d \nu \nu T_{\eta}(\nu \mid \phi),
\end{gathered}
$$

where

$$
\begin{gathered}
S_{\eta}(\nu \mid \phi)=\frac{S_{\eta}(\nu, \phi)}{\int d \nu S_{\eta}(\nu, \phi)}, \\
T_{\eta}(\nu \mid \phi)=\frac{T_{\eta}(\nu, \phi)}{\int d \nu T_{\eta}(\nu, \phi)}
\end{gathered}
$$

are "effective," conditional distributions and where

$$
\begin{aligned}
& S_{\eta}(\phi, \nu)=\int d \phi^{\prime} \Pi_{\phi}\left(\phi^{\prime}\right) S\left(\phi^{\prime}, \nu\right), \\
& T_{\eta}(\phi, \nu)=\int d \phi^{\prime} \Pi_{\phi}\left(\phi^{\prime}\right) T\left(\phi^{\prime}, \nu\right)
\end{aligned}
$$

are "effective" $S$ and $T$ distributions for $\nu$ and $\phi$.

It follows straightforwardly from Eq. (25) that "strange weak values" where the real part of $\nu_{w}$ exceeds the eigenvalue spectrum are only possible for quantum states for which the $T$ distribution in the eigenvalue representation takes negative values. But it is of course well known that classical models may allow observables to exceed the eigenvalue spectrum of the observable. This means also that "strange" weak values may sometimes be supported by a 
classical model. In particular, this may be the case for observables with a discrete spectrum.

The other $c$-number representation that we shall consider is the phase-space representation. In this case, the basis $|\xi\rangle$ should be chosen as eigenstates of the observable canonically conjugate to the postselection observable $\hat{\phi}$. This may provide the possibility of a comparison with a classical phase-space description of weak measurements.

We give a couple of examples illustrating the use of these two representations. First, we consider a weak measurement of a field squared quadrature (or kinetic energy for material particles) $\hat{\nu}=\hat{p}^{2}$ postselected on the canonically conjugate field quadrature (or position for material particles) $\hat{\phi}=\hat{q}$ $[28,29]$. In this case, the eigenvalue representation and the phase-space representation are one and the same, since $\hat{q}$ and $\hat{p}$ are canonically conjugate variables. From Eq. (19) follows that both the eigenvalue representation and the phase-space representation of $\hat{p}^{2}$ postselected on $\hat{q}$ are

$$
T_{\hat{p}^{2}}(q, p)=p^{2} .
$$

Obviously, $T_{\hat{p}^{2}}(q, p) \geqslant 0$. From Eq. (25) and the classicality assumption $T(q, p) \geqslant 0$ follows the inequality

$$
\operatorname{Re}\left(p_{w}^{2}\right)(q) \geqslant 0 .
$$

In this case, a "strange" negative weak value implies a failure of the classical model where $p^{2}$ takes the positive continuum. It can be noted that a negative weak value of kinetic energy $\hat{p}^{2}$ contradicts all stochastic $c$-number models where kinetic energy takes only non-negative values, even models where the $T$ representation of the kinetic energy may differ from the expression (30).

Consider next a weak measurement of the energy of a harmonic oscillator (we use units so that $\omega=1$ )

$$
\hat{H}=\frac{1}{2}\left(\hat{p}^{2}+\hat{q}^{2}\right)=\hat{n}+\frac{1}{2},
$$

assuming postselection on one of the quadratures (or position for a material particle). In the eigenvalue representation,

$$
T_{\hat{H}}(q, n)=n+\frac{1}{2} .
$$

Obviously, $T_{\hat{H}}(q, n) \geqslant \frac{1}{2}$. In the eigenvalue representation, the $T$ representation of the Hamiltonian is bounded from below by the zero-point energy. From Eq. (25) and by imposing the classicality criterion $T(q, n) \geqslant 0$, we may derive the inequality

$$
\operatorname{Re}\left(H_{w}\right)(q) \geqslant \frac{1}{2} .
$$

Violation of this inequality implies "strange" weak values. It can only take place if the $T$ distribution $T(q, n)$ takes negative values. We can split the strange values into two categories. (i) Outputs of the form $\frac{1}{2} \geqslant \operatorname{Re}\left(H_{w}\right)(q) \geqslant 0$ contradict exclusively those classical stochastic models for which the excitation number takes positive values $n \geqslant 0$ or, equivalently, for which the energy is larger than or equal to $1 / 2$. These outputs do not contradict classical models where the energy takes positive values starting from zero. (ii) On the other hand, outputs of the form $0>\operatorname{Re}\left(H_{w}\right)(q)$ contradict all the classical stochastic models where the energy takes positive values.

In the phase-space representation, a weak measurement of the energy of a harmonic oscillator postselected on one of the quadratures calls for the $T$ representation

$$
T_{\hat{H}}(q, p)=\frac{1}{2}\left(p^{2}+q^{2}\right) .
$$

This representation of harmonic oscillator energy coincides with the classical representation and therefore permits an investigation of the limits of classical models. Obviously we have $T_{\hat{H}}(q, p) \geqslant 0$. By assuming that also the state representation is classical, $T(q, p) \geqslant 0$, one may derive from Eq. (21) the inequality

$$
\operatorname{Re}\left(H_{w}\right)(q) \geqslant 0 .
$$

This inequality should be compared with inequality (34). We may say that the phase-space representation highlights just the second category (ii) above: Violation of inequality (36) rules out all the classical stochastic models where the energy takes positive values. Note that the probability of infringing inequality (36) and the probability of the second category (ii) above are equal, since the probability of $\operatorname{Re}\left(H_{w}\right)(q)<0$ does not depend on the representation. On the other hand, the probability that $\operatorname{Re}\left(H_{w}\right)(q)<0$ is less than the probability of $\operatorname{Re}\left(H_{w}\right)(q)<1 / 2$ in agreement with the fact that the first one excludes a larger class of classical models.

We have not mentioned the correspondence principle here. Our purpose is to investigate under what conditions quantum mechanics can be reproduced by a classical stochastic theory. This is not related directly to the classical limit of quantum mechanics.

\section{WEAK MEASUREMENT OF THE PHOTON NUMBER}

The realization of weak measurements requires the coupling of the system to be observed with auxiliary degrees of freedom. The output of the weak measurement is inferred from measurements carried out on the auxiliary system and on the object system itself. In this work we will consider the weak measurement of two observables with non-negative spectra. The boundedness of the spectra is mandatory in order to reveal the appearance of strange values. These observables are $\hat{\nu}=\hat{p}^{2}$ and the number operator $\hat{\nu}=\hat{a}^{\dagger} \hat{a}$, where $\hat{a}$ $=(\hat{q}+i \hat{p}) / \sqrt{2}$ and $\hat{q}, \hat{p}$, with $[\hat{q}, \hat{p}]=i$ are the standard position and linear momentum or field quadratures. For both examples of $\hat{v}$ we will consider the same postselection strategy given by the measurement of the operator $\hat{\phi}=\hat{q}$.

In this section we propose two simple and feasible schemes for the weak measurement of $\hat{a}^{\dagger} \hat{a}$ conditioned on the measurement of $\hat{q}$ in the field of quantum optics, where $\hat{a}^{\dagger} \hat{a}$ represents the number of photons and $\hat{q}$ is a field quadrature. The two possibilities involve different realizations of the auxiliary system. These are another field mode and twolevel atoms. 


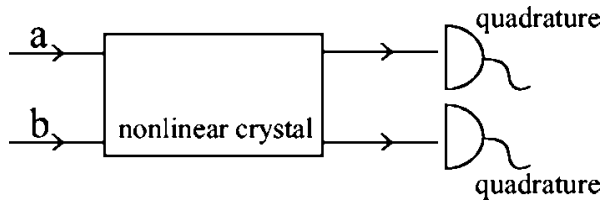

FIG. 1. Scheme for a weak measurement via cross-Kerr coupling in a nonlinear crystal.

\section{A. Coupling to a field mode}

Let us assume that the auxiliary system is another field mode with complex amplitude operator $\hat{b}$. A suitable coupling between the system $\hat{a}$ and the auxiliary variables allowing a weak measurement of the number operator $\hat{a}^{\dagger} \hat{a}$ is of the form

$$
H_{\epsilon}=\epsilon \hat{a}^{\dagger} \hat{a} \hat{b}^{\dagger} \hat{b} .
$$

This coupling can be achieved in practice by propagation of both field modes in crystals with nonlinear optical properties (cross-Kerr interaction) so that $\epsilon$ is proportional to the nonlinear susceptibility of the medium and the length of the crystal. This coupling causes a phase shift of the mode $\hat{b}$ proportional to the photon number in mode $\hat{a}$, which can be then detected simply by measuring a quadrature of the mode $\hat{b}$ (homodyne detection) [30,31]. This example has the advantage that nonlinear effects are usually very weak, so that the requirement $\epsilon \rightarrow 0$ for a weak measurement is naturally satisfied. In Fig. 1 we outline the scheme of the weak measurement of photon number via cross-Kerr coupling conditioned on a quadrature measurement.

\section{B. Coupling to a two-level atom}

The weak measurement of the photon number can also be carried out by coupling the field mode to a two-level atom with internal energy levels $| \pm\rangle$. If the frequency of the field and the resonant frequency of the atom are detuned enough, the atom-field interaction Hamiltonian becomes [30,31]

$$
H_{\epsilon}=\epsilon \hat{a}^{\dagger} \hat{a} \sigma_{z},
$$

where $\sigma_{z}=|+\rangle\langle+|-|-\rangle\langle-|$. In this case we have that $\epsilon$ is inversely proportional to the detuning, so the condition $\epsilon \rightarrow 0$ can be easily achieved.

The atom-field interaction causes a phase shift of the coefficients of an atomic superposition of the states $| \pm\rangle$. The shift is proportional to the photon number and can be detected by measuring, for example, the observable

$$
\sigma_{x}=|+\rangle\langle-|+|+\rangle\langle-| \text {. }
$$

The measurement of $\sigma_{x}$ can be carried out by determining the population of the levels $| \pm\rangle$ after applying to the atom a resonant pulse transforming the eigenstates $| \pm\rangle_{x}$ of $\sigma_{x}$ into $| \pm\rangle$.

The condition of null current density (B9) becomes in this case

$$
{ }_{x}\left\langle \pm\left|\left(\sigma_{z} \rho_{a}+\rho_{a} \sigma_{z}\right)\right| \pm\right\rangle_{x}=0,
$$

which is verified always provided that

$$
\rho_{a}=\frac{1}{2}\left(1+s_{x} \sigma_{x}+s_{y} \sigma_{y}\right)
$$

where $s_{x}, s_{y}$ are real constants such that $s_{x}^{2}+s_{y}^{2} \leqslant 1$ and

$$
\sigma_{y}=i(|+\rangle\langle-|-|+\rangle\langle-|) .
$$

Besides quantum optics, this scheme can be also implemented in the context of trapped ions, where $\hat{a}$ would represent the complex amplitude of the one-dimensional harmonic motion of the center of mass of the trapped ion and $| \pm\rangle$ are two internal levels of the same ion.

\section{COHERENT STATES}

In this section, we turn to the study of weak measurements on coherent states, taking into consideration the effect of thermal noise and finite detector efficiency. It is of particular interest to study the possible nonclassical properties of coherent states, since coherent states are the only pure states that satisfy the Glauber criterion of possessing a nonnegative $P$ distribution.

We shall consider the thermalized coherent state (also known as the displaced thermal state)

$$
\hat{\rho}=\hat{D}(\alpha) \hat{\rho}_{t h} \hat{D}^{\dagger}(\alpha) .
$$

Here $\hat{\rho}_{t h}$ is the density operator for the thermal state and $\hat{D}(\alpha)$ is the displacement operator. This state has a nonnegative $P$ distribution [32]

$$
P(\gamma)=\frac{1}{\pi n_{t h}} e^{-|\gamma-\alpha|^{2} / n_{t h}},
$$

where $\alpha$ is the coherent amplitude when $n_{t h}$ vanishes and $n_{t h}$ is the expected thermal photon number when $\alpha$ vanishes. Since the $P$ distribution is non-negative, this state is essentially classical according to the Glauber criterion.

In this section, we consider weak measurements with postselection on position. The phase space for this experiment consists of position and momentum. In the phase-space representation, the $S$ distribution for this state is

$$
S(q, p)=S_{t h}\left(q-\alpha_{r}, p-\alpha_{i}\right),
$$

where $\alpha=\left(\alpha_{r}+i \alpha_{i}\right) / \sqrt{2}$,

$$
S_{t h}(q, p)=\frac{\exp \left[-\frac{2 \sigma_{t h}^{2}\left(p^{2}+q^{2}\right)-2 i p q}{1+4 \sigma_{t h}^{4}}\right]}{\pi \sqrt{1+4 \sigma_{t h}^{4}}}
$$

is the $S$ distribution of a thermal state [33], and

$$
\sigma_{t h}^{2}=n_{t h}+\frac{1}{2}
$$

is the variance of each quadrature for the thermal distribution. Clearly, the $T$ distribution for this state takes negative values. It is worth emphasizing that the lack of positivity persists for every $n_{t h}$, in sharp contrast to the case of $s$-ordered distributions for which there is always a value of $n_{t h}$ that renders the distribution positive. 
In the following, we assume an imperfect measurement of the operator $\hat{q}$ represented by a Gaussian postselection POVM [see also Eq. (B5)]

$$
\Pi_{q}\left(q^{\prime}\right)=\frac{1}{\sqrt{2 \pi} \sigma_{\eta}} e^{-\left(q-q^{\prime}\right)^{2} /\left(2 \sigma_{\eta}^{2}\right)},
$$

where the width $\sigma_{\eta}$ is determined by detector efficiencies. For example, for a homodyne detector, $\sigma_{\eta}^{2}=(1-\eta) /(2 \eta)$, where $\eta$ is the quantum efficiency of a single detector. We may then derive an effective marginal distribution for the postselection observable:

$$
\rho_{\eta}(q)=\operatorname{Tr}\left[\hat{\Pi}_{q} \hat{\rho}_{s}\right]=\frac{e^{-\left(q-\alpha_{r}\right)^{2} /\left[2\left(\sigma_{t h}^{2}+\sigma_{\eta}^{2}\right)\right]}}{\sqrt{2 \pi\left(\sigma_{t h}^{2}+\sigma_{\eta}^{2}\right)}} .
$$

\section{A. Negative weak value of the kinetic energy}

In this subsection, we consider weak measurements of the observable $\hat{p}^{2}$, which is essentially the kinetic energy [28]. The weak value of $\hat{p}^{2}$ conditioned on the measurement of $\hat{q}$ was found to take negative values for coherent states in Ref. [29]. Here, this treatment will be generalized to include the effects of thermal noise and imperfect detectors.

Since we are considering weak measurements of $\hat{p}^{2}$, the phase-space representation and the eigenvalue representation of the $S$ distribution coincide. It is then useful to define an effective $S$ distribution by using Eqs. (28) and (46). The result is

$$
S_{\eta}(q, p)=S_{\eta}^{t h}\left(q-\alpha_{r}, p-\alpha_{i}\right)
$$

with

$$
S_{\eta}^{t h}(q, p)=\frac{\exp \left[-\frac{2 \sigma_{t h}^{2}\left(p^{2}+q^{2}\right)+2 p^{2} \sigma_{\eta}^{2}-2 i p q}{1+4 \sigma_{t h}^{4}+4 \sigma_{t h}^{2} \sigma_{\eta}^{2}}\right]}{\pi \sqrt{1+4 \sigma_{t h}^{4}+4 \sigma_{t h}^{2} \sigma_{\eta}^{2}}}
$$

being the effective $S$ distribution. Using Eqs. (25) and (51) we find that

$$
\operatorname{Re}\left[\left(p^{2}\right)_{w}\right]=\frac{1+4\left(\alpha_{i}^{2}+\sigma_{t h}^{2}\right)\left(\sigma_{t h}^{2}+\sigma_{\eta}^{2}\right)}{4\left(\sigma_{t h}^{2}+\sigma_{\eta}^{2}\right)}-\frac{\left(q-\alpha_{r}\right)^{2}}{4\left(\sigma_{t h}^{2}+\sigma_{\eta}^{2}\right)^{2}} .
$$

The roots of this polynomial are

$$
q_{ \pm}=\alpha_{r} \pm \sqrt{\left(\sigma_{t h}^{2}+\sigma_{\eta}^{2}\right)\left[1+4\left(\alpha_{i}^{2}+\sigma_{t h}^{2}\right)\left(\sigma_{t h}^{2}+\sigma_{\eta}^{2}\right)\right]} .
$$

The probability of postselecting a position $q$ which on average gives a negative weak value of $\hat{p}^{2}$ then is

$$
P\left[\operatorname{Re}\left(p^{2}\right)_{w}<0\right]=1-\int_{q_{-}}^{q_{+}} d q \rho_{\eta}(q) .
$$

The result is

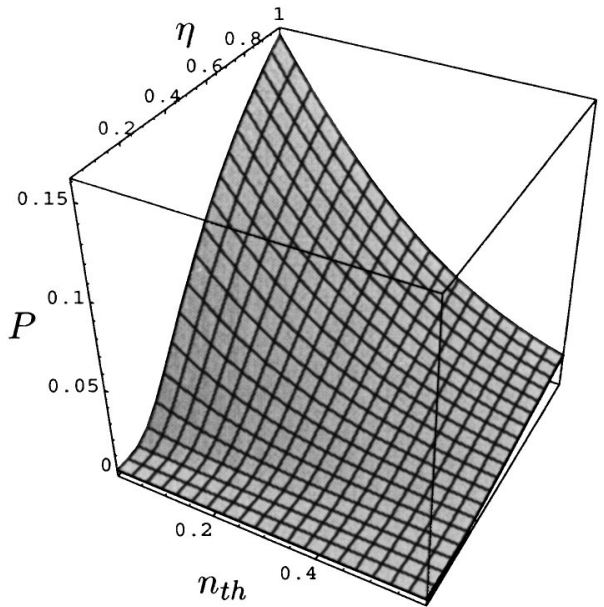

FIG. 2. The probability for observing a negative weak value of $\hat{p}^{2}$ as a function of the detector efficiency $\eta$ and the average thermal occupation number $n_{t h}$. It is assumed that $\alpha_{i}=0$, whereas $\alpha_{r}$ may take any value. This effect therefore is present even for macroscopic excitations $\alpha_{r}$.

$$
P\left[\operatorname{Re}\left(p^{2}\right)_{w}<0\right]=\operatorname{erfc} \sqrt{\frac{1}{2}+2\left(\alpha_{i}^{2}+\sigma_{t h}^{2}\right)\left(\sigma_{t h}^{2}+\sigma_{\eta}^{2}\right)} .
$$

The complementary error function $\operatorname{erfc}(x)$ is monotonically decreasing. Therefore, this probability is maximized when $\alpha_{i}^{2}, \sigma_{t h}^{2}$, and $\sigma_{\eta}^{2}$ are as small as possible. The state can be chosen so that $\alpha_{i}=0$. For a perfect detector $\sigma_{\eta}=0$. The quadrature variance $\sigma_{t h}^{2}$ is bounded below by $1 / 2$. Therefore, the maximum probability for a negative weak value of $\hat{p}^{2}$ is erfc $1 \approx 0.16$ [29]. It is particularly interesting to note that the effect is independent of the real part of the amplitude, $\alpha_{r}$. A negative weak value therefore can be observed for a macroscopic occupation of the mode.

This probability has been plotted as a function of detector efficiency $\eta$ and thermal occupation number $n_{t h}$ in Fig. 2. As noted in Sec. IV, the negativity of $\operatorname{Re}\left(p_{w}^{2}\right)$ contradicts all classical stochastic models. A weak value of the kinetic energy might be observed for material particles at low temperatures. However, a realizable quantum optical experiment is not known to the authors. To investigate a feasible quantum optical experiment, we turn to the weak measurement of photon number and energy.

\section{B. Negative weak value of the energy}

In this subsection we study weak measurements of energy conditioned on the postselection of a quadrature observable. As demonstrated in Sec. IV, a negative weak value of the energy for a harmonic oscillator contradicts classical stochastic models.

By combining Eqs. (21), (46), and (48) we find that

$$
\operatorname{Re}\left[H_{w}(q)\right]=a q^{2}+b q+c,
$$

where 


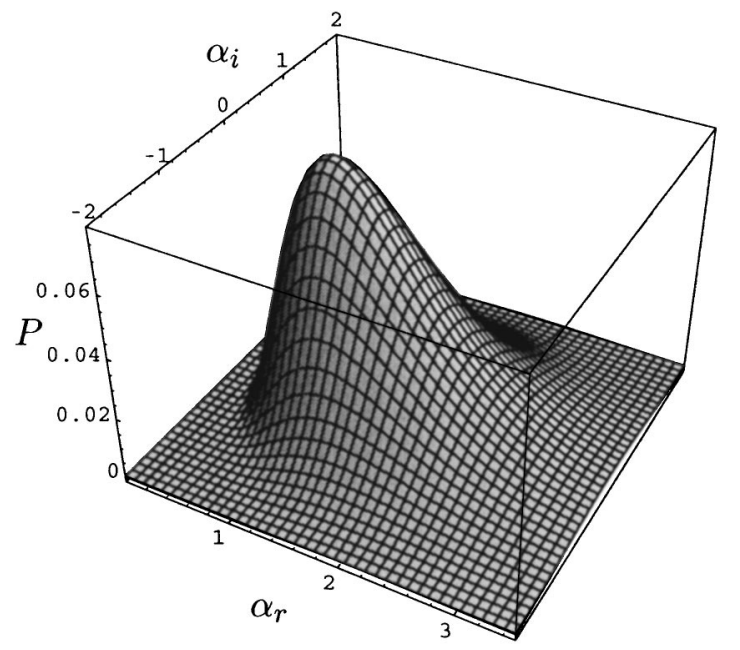

FIG. 3. The probability for observing a negative weak value of energy as a function of the coherent state amplitudes $\alpha_{r}$ and $\alpha_{i}$. We assume perfect detectors $\sigma_{\eta}=0$ and no thermal noise $n_{t h}=0$. The probability has a maximum at $\alpha_{r}=1, \alpha_{i}=0$, at which the probability is $(1 / 2)$ erfc $1 \approx 0.079$.

$$
\begin{gathered}
a=\frac{4 \sigma_{t h}^{4}-1}{8\left(\sigma_{t h}^{2}+\sigma_{\eta}^{2}\right)^{2}}, \\
b=\frac{\alpha_{r}\left(4 \sigma_{t h}^{2} \sigma_{\eta}^{2}+1\right)}{4\left(\sigma_{t h}^{2}+\sigma_{\eta}^{2}\right)^{2}}, \\
c=\frac{\sigma_{t h}^{2}}{2}+\frac{\alpha_{i}^{2}}{2}+\frac{1+4 \sigma_{t h}^{2} \sigma_{\eta}^{2}}{8\left(\sigma_{t h}^{2}+\sigma_{\eta}^{2}\right)}+\frac{\alpha_{r}^{2}\left(4 \sigma_{\eta}^{4}-1\right)}{8\left(\sigma_{t h}^{2}+\sigma_{\eta}^{2}\right)^{2}} .
\end{gathered}
$$

Two real roots $q_{ \pm}$exist provided that $b^{2} \geqslant 4 a c$ (only one if $a=0)$. This establishes a necessary condition to be fulfilled by $\sigma_{t h}, \sigma_{\eta}, \alpha_{r}$, and $\alpha_{i}$ for the existence of negative values for $\operatorname{Re}\left[H_{w}(q)\right]$. If this is satisfied, the probability of observing a negative $\operatorname{Re}\left(H_{w}\right)$ is

$$
P\left[\operatorname{Re}\left(H_{w}\right)<0\right]=\int_{Q_{-}}^{Q_{+}} d q \rho_{\eta}(q) .
$$

For ideal detectors $\sigma_{\eta}=0$ and vanishing thermal noise $n_{t h}$ $=0$ this probability can be written as

$$
P\left[\operatorname{Re}\left(H_{w}\right)<0\right]=\frac{1}{2} \operatorname{erfc}\left(\frac{1+\alpha_{r}^{2}+\alpha_{i}^{2}}{2\left|\alpha_{r}\right|}\right) .
$$

This probability has been plotted in Fig. 3. It reaches a maximum at $\alpha_{r}=1$ and $\alpha_{i}=0$, at which the probability is $(1 / 2)$ erfc $1 \approx 0.08$. This is half of the probability for observing a negative weak value of kinetic energy.

A more complex expression for the probability can be obtained for arbitrary detector efficiency and thermal excitation. This has been plotted in Fig. 4. We see that $\alpha_{r}$ now must reach a minimum value in order to see nonclassical negative weak values. In Fig. 5, the probability is plotted as a function of detector efficiency $\eta$ and average thermal occupation number $n_{t h}$.

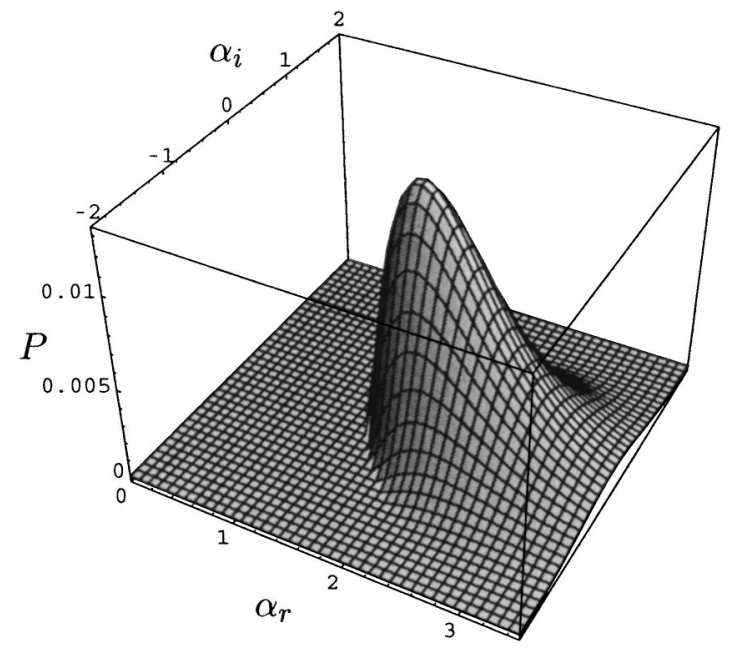

FIG. 4. The probability for observing a negative weak value of energy as a function of the coherent state amplitudes $\alpha_{r}$ and $\alpha_{i}$. We assume detectors with quantum efficiency $\eta=0.7$ and thermal occupation number $n_{t h}=0.3$. There is a minimum $\alpha_{r}$ required in order to see nonclassical behavior.

The negativity of weak values of the kinetic energy and of the total energy both contradict a classical, stochastic model of light. Negative weak values of the kinetic energy persist also for macroscopic coherent amplitudes. This does not occur for a weak value of the energy. On the other hand, a weak measurement of the energy has a feasible practical measuring scheme as outlined in Sec. V.

\section{Negative weak value of the photon number}

It was demonstrated in Sec. IV that a negative weak value of the photon number contradicts a classical stochastic model where the energy is bounded from below by the zero-point energy. We study this further here.

The weak value of the photon number is simply

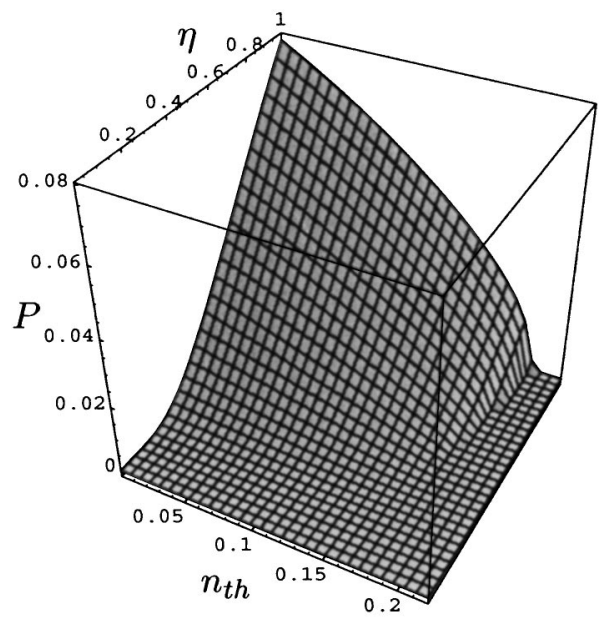

FIG. 5. The probability for observing a negative weak value of energy as a function of the detector efficiency $\eta$ and the average thermal occupation number $n_{t h}$. The coherent excitation is assumed to be $\alpha_{r}=1, \alpha_{i}=0$. 


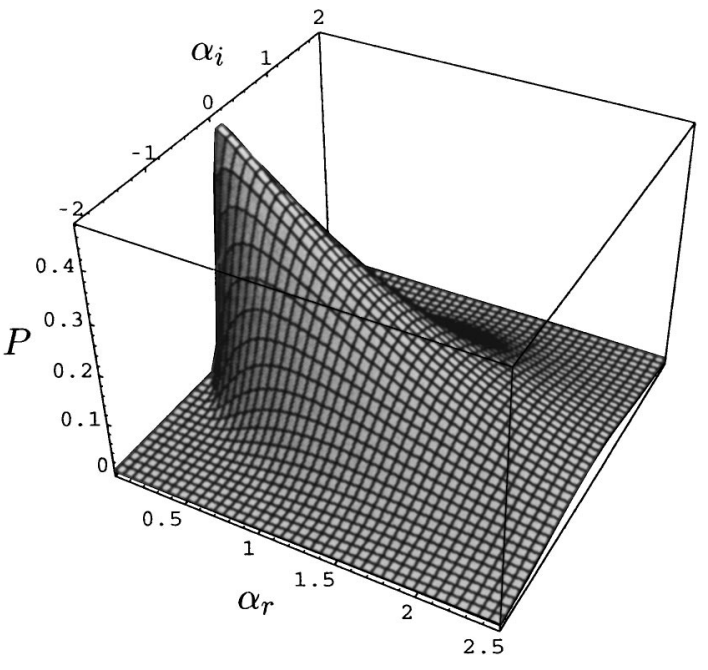

FIG. 6. The probability for observing a negative weak value of the photon number $\hat{n}$ as a function of the coherent-state amplitudes $\alpha_{r}$ and $\alpha_{i}$. We assume perfect detectors $\sigma_{\eta}=0$ and no thermal noise $n_{t h}=0$. The probability increases with decreasing $|\alpha|$, but has a singularity at $|\alpha|=0$.

$$
n_{w}(q)=H_{w}(q)-\frac{1}{2}
$$

Two real roots $q_{ \pm}$of $n_{w}$ exist provided that $b^{2} \geqslant 4 a(c-1 / 2)$. In the case that this condition is satisfied the probability of observing a negative $\operatorname{Re}\left[n_{w}(q)\right]$ is

$$
P\left[\operatorname{Re}\left(n_{w}\right)<0\right]=\int_{q_{-}}^{q_{+}} d q \rho_{\eta}(q) .
$$

For ideal detectors and vanishing thermal noise this probability can be written as

$$
P\left[\operatorname{Re}\left(n_{w}\right)<0\right]=\frac{1}{2} \operatorname{erfc}\left(\frac{\alpha_{r}^{2}+\alpha_{i}^{2}}{2\left|\alpha_{r}\right|}\right) .
$$

This function has been plotted in Fig. 6. The probability is always maximized by letting $\alpha_{i} \rightarrow 0$. It approaches a maximum of 0.5 for vanishing $\alpha_{r}$. However, it has a singularity in $\alpha_{r}=0$ and actually vanishes in this point. Thus, there is zero probability of observing a negative weak value of $\hat{n}$ for the vacuum state. There must be a finite small coherent amplitude to see this.

A more complex expression is obtained for finite detector efficiency and finite thermal noise. The probability has been plotted in this case in Fig. 7. We see that $\alpha_{r}$ now must reach a minimum value in order to see nonclassical negative weak values.

The probability has been plotted as a function of detector efficiency $\eta$ and the average thermal occupation number $n_{t h}$ in Fig. 8. We see that for finite thermal occupation number $n_{t h}$ there is a lower bound on the detector efficiency $\eta$ to see nonclassical behavior. This bound vanishes when $n_{t h} \rightarrow 0$.

\section{CONCLUSIONS}

In this work we have examined a very general form of weak measurements focusing on the emergence of nonclas-

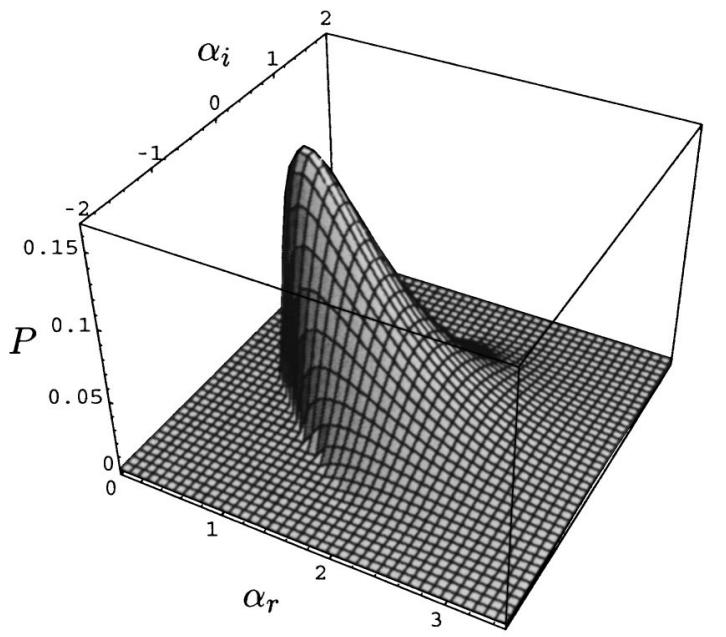

FIG. 7. The probability for observing a negative weak value of the photon number $\hat{n}$ as a function of the coherent-state amplitudes $\alpha_{r}$ and $\alpha_{i}$. We assume detectors with quantum efficiency $\eta=0.7$ and thermal occupation number $n_{t h}=0.3$. We see that there is a minimum $\alpha_{r}$ required in order to see nonclassical behavior.

sical features. We have shown that the appearance of strange weak values is equivalent to the existence of negative values for a generalized Terletsky-Margenau-Hill distribution.

We have presented some feasible practical implementations of this kind of measurement in the field of quantum optics focusing on the weak measurement of photon number and energy. We have demonstrated that negative weak values of the energy contradict all classical models of light and that negative weak values of the photon number contradict a classical model where the energy is bounded from below by the zero-point energy.

As a particular but striking enough example we have considered weak measurements of coherent states. We have

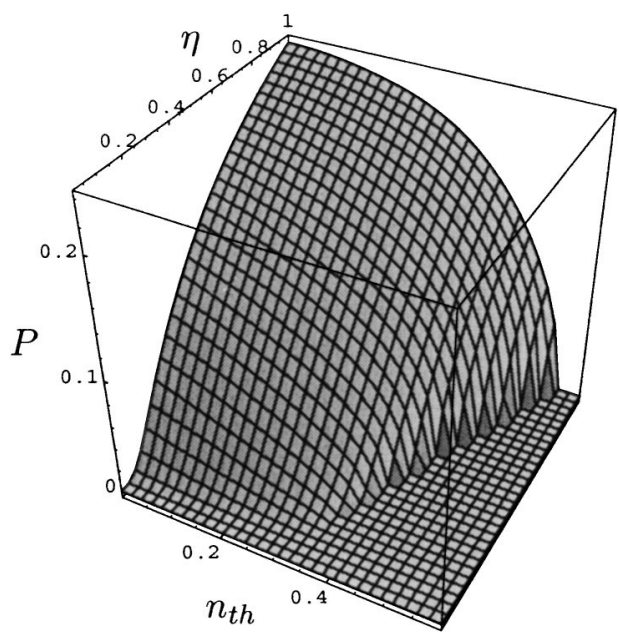

FIG. 8. The probability for observing a negative weak value of the photon number $\hat{n}$ as a function of detector efficiency $\eta$ and average thermal occupation number $n_{t h}$. The coherent excitation is assumed to be $\alpha_{r}=0.1, \alpha_{i}=0$. A minimum detector efficiency (and a maximum thermal occupation number) is required to observe a negative weak value. 
found that negative weak values can be observed with a probability of $16 \%$ for the kinetic energy (or squared field quadrature), $8 \%$ for the harmonic oscillator energy, and 50\% for the photon number.

We have analyzed the persistence of the effect under practical experimental conditions by considering degrading imperfections such as the presence of thermal fluctuations and the use of inefficient detectors. All these results confirm the possibility of a practical observation of nonclassical effects for states previously considered as firm examples of classical behavior.

\section{APPENDIX A: IMPERFECT DETECTORS}

An imperfect detector may be represented by a positiveoperator-valued measure. To represent imperfect detection of an observable $\hat{\phi}$, we study the class of diagonal POVM's

$$
\hat{\Pi}_{\phi}=\int d \phi^{\prime} \Pi_{\phi}\left(\phi^{\prime}\right)\left|\phi^{\prime}\right\rangle\left\langle\phi^{\prime}\right|,
$$

where $|\phi\rangle$ are eigenstates of $\hat{\phi}$. These states are assumed to constitute a complete set:

$$
\int d \phi|\phi\rangle\langle\phi|=\mathrm{I} .
$$

The diagonal form is assumed because interference between different detector states should not occur. We assume that $\Pi_{\phi}\left(\phi^{\prime}\right)$ is a non-negative function.

The POVM should provide a resolution of the identity operator

$$
\int d \phi \hat{\Pi}_{\phi}=\mathrm{I}
$$

This implies that

$$
\int d \phi \Pi_{\phi}\left(\phi^{\prime}\right)=1
$$

Thus, $\Pi_{\phi}\left(\phi^{\prime}\right)$ should be a normalized distribution over $\phi$. The probability distribution for the observable $\hat{\phi}$, taking into account the imperfect detector represented by $\Pi_{\phi}$, is

$$
\operatorname{Tr}\left[\hat{\Pi}_{\phi} \hat{\rho}\right]=\int d \phi^{\prime} \Pi_{\phi}\left(\phi^{\prime}\right)\left\langle\phi^{\prime}|\hat{\rho}| \phi^{\prime}\right\rangle .
$$

We require that the imperfect detector should give the same expected reading as a perfect detector. This condition of unbiasedness can be written as

$$
\int d \phi \phi \operatorname{Tr}\left[\hat{\Pi}_{\phi} \hat{\rho}\right]=\int d \phi \phi\langle\phi|\hat{\rho}| \phi\rangle,
$$

and it implies that

$$
\phi^{\prime}=\int d \phi \phi \Pi_{\phi}\left(\phi^{\prime}\right)
$$

Thus, unbiasedness is equivalent to requiring that the parameter $\phi^{\prime}$ should be the expectation value of the distribution $\Pi_{\phi}$.
A typical diagonal POVM satisfying the properties (A4) and (A7) is the Gaussian

$$
\Pi_{\phi}\left(\phi^{\prime}\right)=\frac{1}{\sqrt{2 \pi \sigma^{2}}} e^{-\left(\phi-\phi^{\prime}\right)^{2} /\left(2 \sigma^{2}\right)} .
$$

For this POVM and for the subset of POVM's where $\Pi_{\phi}\left(\phi^{\prime}\right)$ is a function of $\left(\phi-\phi^{\prime}\right)$ only, the transformation (A5) is a convolution. However, in the most general case the POVM does not depend on $\left(\phi-\phi^{\prime}\right)$ only. For example, the conditions (A4) and (A7) are also satisfied by all Gaussian POVM's of the type (A8) even when the standard deviation $\sigma$ is an arbitrary function of $\phi^{\prime}$.

\section{APPENDIX B: DERIVATION OF WEAK VALUES}

Prior to the measurement interaction, we assume that the object is in the state $\hat{\rho}_{s}$ and the pointer is in an arbitrary mixed state $\hat{\rho}_{a}$. The total density operator prior to the interaction has the product form $\hat{\rho}_{0}=\hat{\rho}_{s} \otimes \hat{\rho}_{a}$.

After the interaction, the total density operator has evolved to

$$
\hat{\rho}_{\epsilon}=\hat{U}_{\epsilon} \hat{\rho}_{0} \hat{U}_{\epsilon}^{\dagger},
$$

where $\hat{U}_{\epsilon}$ is a unitary evolution operator. Since the Hamiltonian does not depend explicitly on time, we can write

$$
\hat{U}_{\epsilon}=e^{-i \int \hat{H} d t}=e^{-i \epsilon \hat{\nu} \otimes \hat{P}} .
$$

We may expand this evolution operator to first order in $\epsilon$ as

$$
\hat{U}_{\epsilon} \approx 1-i \epsilon \hat{\nu} \otimes \hat{P} .
$$

To first order in $\epsilon$, the density operator after the interaction then can be written as

$$
\hat{\rho}_{\epsilon}=\hat{\rho}_{0}+i \epsilon\left[\hat{\rho}_{0}, \hat{\nu} \otimes \hat{P}\right] .
$$

After the interaction, we assume that imperfect measurements are made of the pointer observable $\hat{Q}$ and the object observable $\hat{\phi}$. Each observable is represented by a diagonal POVM

$$
\begin{aligned}
& \hat{\Pi}_{\phi}=\int d \phi^{\prime} \Pi_{\phi}\left(\phi^{\prime}\right)\left|\phi^{\prime}\right\rangle\left\langle\phi^{\prime}\right|, \\
& \hat{\Pi}_{Q}=\int d Q^{\prime} \Pi_{Q}\left(Q^{\prime}\right)\left|Q^{\prime}\right\rangle\left\langle Q^{\prime}\right| .
\end{aligned}
$$

Both $\Pi_{\phi}$ and $\Pi_{Q}$ are assumed to be classical distributions. In this way, the detectors are assumed to be in a statistical mixture of pure projector states. We also have assumed that the observable $\phi$ has a continuous spectrum. There is no loss of generality in this.

The joint probability density for $\phi$ and $Q$ after the interaction is

$$
\rho_{\epsilon}(\phi, Q)=\operatorname{Tr}\left(\hat{\Pi}_{\phi} \hat{\Pi}_{Q} \hat{\rho}_{\epsilon}\right),
$$

where the trace is taken over both $\mathcal{H}_{s}$ and $\mathcal{H}_{a}$. Using the expression (B4) for the first-order density operator, we find that 


$$
\begin{aligned}
\rho_{\epsilon}(\phi, Q)= & \operatorname{Tr}\left(\hat{\Pi}_{\phi} \hat{\rho}_{s}\right) \operatorname{Tr}\left(\hat{\Pi}_{Q} \hat{\rho}_{a}\right)+i \epsilon \operatorname{Tr}\left(\hat{\Pi}_{\phi} \hat{\Pi}_{Q}\left[\hat{\rho}_{0}, \hat{\nu} \otimes \hat{P}\right]\right) \\
= & \operatorname{Tr}\left(\hat{\Pi}_{\phi} \hat{\rho}_{s}\right) \operatorname{Tr}\left(\hat{\Pi}_{Q} \hat{\rho}_{a}\right)+i \epsilon\left[\operatorname{Tr}\left(\hat{\Pi}_{\phi} \hat{\rho}_{s} \hat{\nu}\right) \operatorname{Tr}\left(\hat{\Pi}_{Q} \hat{\rho}_{a} \hat{P}\right)\right. \\
& \left.-\operatorname{Tr}\left(\hat{\Pi}_{\phi} \hat{\nu} \hat{\rho}_{s}\right) \operatorname{Tr}\left(\hat{\Pi}_{Q} \hat{P} \hat{\rho}_{a}\right)\right] .
\end{aligned}
$$

We require that the current density of the pointer vanish:

$$
\left\langle Q\left|\left(\hat{P} \hat{\rho}_{a}+\hat{\rho}_{a} \hat{P}\right)\right| Q\right\rangle=0 .
$$

This implies that

$$
\operatorname{Tr}\left[\hat{\Pi}_{Q}\left(\hat{P} \hat{\rho}_{a}+\hat{\rho}_{a} \hat{P}\right)\right]=0 .
$$

Therefore we can write

$$
\begin{aligned}
\rho_{\epsilon}(\phi, Q)= & \operatorname{Tr}\left(\hat{\Pi}_{\phi} \hat{\rho}_{s}\right) \operatorname{Tr}\left(\hat{\Pi}_{Q} \hat{\rho}_{a}\right) \\
& +i \epsilon \operatorname{Tr}\left[\hat{\Pi}_{\phi}\left(\hat{\rho}_{s} \hat{\nu}+\hat{\nu} \hat{\rho}_{s}\right)\right] \operatorname{Tr}\left(\hat{\Pi}_{Q} \hat{\rho}_{a} \hat{P}\right) .
\end{aligned}
$$

The marginal distribution for the object observable $\phi$ after the interaction is

$$
\rho_{\epsilon}(\phi)=\int d Q \rho_{\epsilon}(\phi, Q) .
$$

Due to the vanishing of the current density of the probe, it is found that

$$
\rho_{\epsilon}(\phi)=\operatorname{Tr}\left(\hat{\Pi}_{\phi} \hat{\rho}_{s}\right) .
$$

Therefore, the probability distribution for the postselection observable $\phi$ is unaffected by the measurement interaction. Note that we have considered an arbitrary postselection measurement. This means in fact that the probability distribution for every possible object observable is unaffected by the measurement interaction.

The conditional probability density for the pointer position $Q$ given the postselection outcome $\phi$ is defined as

$$
\rho(Q \mid \phi)=\frac{\rho_{\epsilon}(\phi, Q)}{\rho_{\epsilon}(\phi)} .
$$

We find that

$$
\rho(Q \mid \phi)=\operatorname{Tr}\left(\hat{\Pi}_{Q} \hat{\rho}_{a}\right)+2 i \epsilon \operatorname{Re}\left(\nu_{w}\right) \operatorname{Tr}\left(\hat{\Pi}_{Q} \hat{\rho}_{a} \hat{P}\right),
$$

where

$$
\nu_{w}(\phi)=\frac{\operatorname{Tr}\left(\hat{\Pi}_{\phi} \hat{\nu} \hat{\rho}_{s}\right)}{\operatorname{Tr}\left(\hat{\Pi}_{\phi} \hat{\rho}_{s}\right)}
$$

is the weak value of $\hat{\nu}$ for an unsharp postselection represented by the POVM $\hat{\Pi}_{\phi}$. Using Eq. (B9) it can be shown that

$$
\left\langle Q\left|\hat{\rho}_{a} \hat{P}\right| Q\right\rangle=\frac{i}{2} \frac{\partial}{\partial Q}\left\langle Q\left|\hat{\rho}_{a}\right| Q\right\rangle .
$$

Hence we can write

$$
\begin{aligned}
\operatorname{Tr}\left(\hat{\Pi}_{Q} \hat{\rho}_{a} \hat{P}\right) & =\int d Q^{\prime \prime} \int d Q^{\prime} \Pi_{Q}\left(Q^{\prime}\right)\left\langle Q^{\prime \prime} \mid Q^{\prime}\right\rangle\left\langle Q^{\prime}\left|\hat{\rho}_{a} \hat{P}\right| Q^{\prime \prime}\right\rangle \\
& =\int d Q^{\prime} \Pi_{Q}\left(Q^{\prime}\right)\left\langle Q^{\prime}\left|\hat{\rho}_{a} \hat{P}\right| Q^{\prime}\right\rangle \\
& =\frac{i}{2} \int d Q^{\prime} \Pi_{Q}\left(Q^{\prime}\right) \frac{\partial}{\partial Q^{\prime}}\left\langle Q^{\prime}\left|\hat{\rho}_{a}\right| Q^{\prime}\right\rangle \\
& =-\frac{i}{2} \int d Q^{\prime} \frac{\partial \Pi_{Q}\left(Q^{\prime}\right)}{\partial Q^{\prime}}\left\langle Q^{\prime}\left|\hat{\rho}_{a}\right| Q^{\prime}\right\rangle,
\end{aligned}
$$

where we have assumed that $\left\langle Q^{\prime}\left|\hat{\rho}_{a}\right| Q^{\prime}\right\rangle$ vanishes at the integration borders.

The conditional probability density for the pointer position $Q$ given the outcome $\phi$ of the postselection is

$$
\rho_{\epsilon}(Q \mid \phi)=\int d Q^{\prime} \Pi_{Q}\left(Q^{\prime}+\epsilon \operatorname{Re} \nu_{w}\right)\left\langle Q^{\prime}\left|\hat{\rho}_{a}\right| Q^{\prime}\right\rangle,
$$

where

$$
\Pi_{Q}\left(Q^{\prime}+\epsilon \operatorname{Re} \nu_{w}\right)=\left[1+\epsilon \operatorname{Re}\left(\nu_{w}\right) \frac{\partial}{\partial Q^{\prime}}\right] \Pi_{Q}\left(Q^{\prime}\right) .
$$

We introduce the expectation value of the pointer position conditioned on the postselection outcome:

$$
E_{\epsilon}(Q \mid \phi)=\int d Q Q \rho_{\epsilon}(Q \mid \phi) .
$$

By using Eqs. (B19) and (B20) we find that

$$
\begin{aligned}
E_{\epsilon}(Q \mid \phi)= & E_{0}(Q \mid \phi)+\epsilon \operatorname{Re}\left(\nu_{w}\right) \\
& \times \int d Q Q \int d Q^{\prime} \frac{\partial \Pi_{Q}\left(Q^{\prime}\right)}{\partial Q^{\prime}}\left\langle Q^{\prime}\left|\hat{\rho}_{a}\right| Q^{\prime}\right\rangle .
\end{aligned}
$$

A reorganization of terms gives

$$
\begin{aligned}
E_{\epsilon}(Q \mid \phi)= & E_{0}(Q \mid \phi)+\epsilon \operatorname{Re}\left(\nu_{w}\right) \\
& \times \int d Q^{\prime}\left\langle Q^{\prime}\left|\hat{\rho}_{a}\right| Q^{\prime}\right\rangle \frac{\partial}{\partial Q^{\prime}} \int d Q Q \Pi_{Q}\left(Q^{\prime}\right) .
\end{aligned}
$$

We assume that the POVM is unbiased, fulfilling condition (A7). This, together with the normalization property of the position distribution, implies that

$$
E_{\epsilon}(Q \mid \phi)=E_{0}(Q \mid \phi)+\epsilon \operatorname{Re}\left(\nu_{w}\right) .
$$

This shows that the pointer observable $Q$ has been translated a distance $\epsilon \operatorname{Re}\left(\nu_{w}\right)$, and this setup therefore allows for a measurement of $\operatorname{Re}\left(\nu_{w}\right)$. The only restriction on the auxiliary pointer system is that the current density should vanish. 
[1] H. J. Kimble, M. Dagenais, and L. Mandel, Phys. Rev. Lett. 39, 691 (1977).

[2] Y. Aharonov, D. Z. Albert, and L. Vaidman, Phys. Rev. Lett. 60, 1351 (1988).

[3] L. M. Johansen, Phys. Rev. Lett. 93, 120402 (2004).

[4] J. G. Kirkwood, Phys. Rev. 44, 31 (1933).

[5] C. L. Mehta, J. Math. Phys. 5, 677 (1964).

[6] Y. P. Terletsky, Zh. Eksp. Teor. Fiz. 7, 1290 (1937).

[7] H. Margenau and R. N. Hill, Prog. Theor. Phys. 26, 722 (1961).

[8] R. J. Glauber, Phys. Rev. 131, 2766 (1963).

[9] E. C. G. Sudarshan, Phys. Rev. Lett. 10, 277 (1963).

[10] U. M. Titulaer and R. J. Glauber, Phys. Rev. 140, B676 (1965)

[11] M. Hillery, Phys. Lett. 111A, 409 (1985).

[12] L. Mandel and E. Wolf, Optical Coherence and Quantum Optics (Cambridge University Press, New York, 1995).

[13] V. V. Dodonov, J. Opt. B: Quantum Semiclassical Opt. 4, R1 (2002).

[14] M. Hillery, Phys. Rev. A 35, 725 (1987).

[15] C. T. Lee, Phys. Rev. A 44, R2775 (1991).

[16] W. Vogel, Phys. Rev. Lett. 84, 1849 (2000).

[17] T. Richter and W. Vogel, Phys. Rev. Lett. 89, 283601 (2002).
[18] J. von Neumann, Mathematical Foundations of Quantum Mechanics (Princeton University Press, Princeton, 1955).

[19] F. Haake and D. F. Walls, Phys. Rev. A 36, 730 (1987).

[20] A. M. Steinberg, Phys. Rev. A 52, 32 (1995).

[21] A. M. Steinberg, Phys. Rev. Lett. 74, 2405 (1995).

[22] L. Cohen, Phys. Lett. A 212, 315 (1996).

[23] R. I. Sutherland, J. Math. Phys. 23, 2389 (1982).

[24] L. Cohen and C. Lee, Found. Phys. 17, 561 (1987).

[25] J. G. Muga, J. P. Palao, and R. Sala, Phys. Lett. A 238, 90 (1998).

[26] L. M. Johansen, Phys. Lett. A 322, 298 (2004).

[27] A. Luis, Phys. Rev. A 67, 064101 (2003).

[28] Y. Aharonov, S. Popescu, D. Rohrlich, and L. Vaidman, Phys. Rev. A 48, 4084 (1993).

[29] L. M. Johansen, Phys. Lett. A 329, 184 (2004).

[30] D. F. Walls and G. J. Milburn, Quantum Optics (SpringerVerlag, Berlin, 1994).

[31] M. O. Scully and M. S. Zubairy, Quantum Optics (Cambridge University Press, Cambridge, England, 1997).

[32] G. Lachs, Phys. Rev. 138, B1012 (1965).

[33] L. M. Johansen, J. Opt. B: Quantum Semiclassical Opt. 6, L21 (2004). 